\title{
http://revistainvestigacionacademicasinfrontera.com
}

\section{Importancia de la Gestión De Costos en Las PYMES Del Sector Servicio de Hermosillo, Sonora.}

\author{
Martín Guillermo Durán Acosta1 \\ María Lizett Zolano Sánchez2 \\ Leticia Del Carmen Encinas Meléndrez ${ }^{3}$
}

\begin{abstract}
Resumen
La mayoría de los empresarios de Pequeñas y Medianas Empresas (Pymes) determinan el costo de su producto para tener una base para fijar (o negociar) el precio de venta y, además, calculan los resultados de su negocio como diferencia entre sus ingresos y costos totales. Sin embargo, un buen sistema de costos es mucho más que esto, es una poderosa herramienta de planificación, control y gestión que permite identificar las causas reales por las que la empresa incurre en mayores costos. En esta investigación, tomamos como referencia a las Pymes del sector de servicio afiliados a CANACO en la ciudad de Hermosillo, Sonora y registrados en el padrón del SIEM con una fuerza de trabajo entre 10 y 250 trabajadores, un mínimo de diez años en la actividad. El objetivo de esta investigación es determinar la importancia de los costos y sus beneficios en la gestión empresarial de las Pymes. Los principales resultados de la presente investigación, muestran que para la gerencia Pyme del sector servicio afiliadas a CANACO de Hermosillo, Sonora, los costos son generadores de información y que, si se genera de manera oportuna, permite tomar mejores decisiones para establecer estrategias que le ayuden a la empresa a lograr ventaja competitiva. En conclusión, existe la necesidad de implementar en las Pymes, un modelo de costos razonable y apropiado de acuerdo con las necesidades de estas empresas, para ayudar a evitar tomar decisiones erróneas, en la medida en que proporcione información confiable, objetiva y oportuna para la dirección.
\end{abstract}

Palabras Clave: Costos, Estrategia, Ventaja Competitiva, Información.

\footnotetext{
${ }^{1}$ Departamento de Contabilidad, Universidad de Sonora, Unidad Centro. Blvd. Luis Encinas y Rosales $s / n$, Colonia Centro, Hermosillo, Sonora Tel.01(642) 2592211 y 12, correo electrónico: patricia.aguilar@unison.mx

${ }^{2}$ Departamento de Ciencias Administrativas y Agropecuarias, Universidad de Sonora, Unidad Santa Ana. Carr. internacional y Av. 16 de Septiembre, Santa Ana, Sonora, México Tel. 01(641)3241120 correo electrónico: lizoza@ hotmail.com

${ }^{3}$ Departamento de Administración, Universidad de Sonora, Unidad Centro. Blvd. Luis Encinas y Rosales $s / n$, Colonia Centro, Hermosillo, Sonora, México Tel.01(662)259.2168 correo electrónico: leticia.encinas@unison.mx
} 


\begin{abstract}
The majority of Small and Medium Enterprises (SMEs) entrepreneurs determine the cost of their product to have a basis to fix (or negotiate) the sale price and, in addition, calculate the results of their business as a difference between their income and costs. totals However, a good cost system is much more than this, it is a powerful planning, control and management tool that allows to identify the real causes for which the company incurs higher costs. In this research, we take as reference the service sector SMEs affiliated to CANACO in the city of Hermosillo, Sonora and registered in the SIEM registry with a work force of between 10 and 250 workers, a minimum of ten years in the activity. The objective of this research is to determine the importance of costs and their benefits in the business management of SMEs. The main results of the present investigation show that for the SME management of the service sector affiliated with CANACO of Hermosillo, Sonora, the costs are generators of information and that, if this is generated in a timely manner, it allows to make better decisions to establish strategies that help the company achieve competitive advantage. In conclusion, there is a need to implement in SMEs, a reasonable and appropriate costing model according to needs of these companies, to help avoid making wrong decisions, to the extent that it provides reliable, objective and timely information for management.
\end{abstract}

Key words: Key words: Costs, Strategy, Competitive Advantage, Information.

\title{
Introducción
}

La competitividad es un término multidimensional que no posee una definición específica, debido a los diferentes niveles y factores cualitativos y cuantitativos que intervienen en su determinación, asimismo comprende la existencia de diversos enfoques de análisis e indicadores para su medición. Además, es un concepto universal utilizado como fórmula para el crecimiento económico de los países y como condición necesaria cuando se trata de ganar participación en los mercados internacionales (Flores, 2008, citado por Saavedra, Milla y Sánchez, 2013). Martínez, Charterina y Araujo (2010), dentro de su artículo Un Modelo Causal de Competitividad Empresarial, denuestan la validación de la competitividad por medio de los recursos y estrategias internas de la empresa para lograr una ventaja competitiva, dando mayor validez a mi modelo propuesto para eficientar los recursos y alcanzar las metas de la Empresa.

En este tiempo de globalización se observan cambios relevantes en el ámbito económico y financiero y se advierte una fuerte competencia empresarial, manejo de información electrónica 
Año 11.

Revista de Investigación

Núm. 28

Académica sin Frontera

ISSN: 2007-8870

\section{http://revistainvestigacionacademicasinfrontera.com}

de vanguardia y nuevas formas de comercialización (López y Marín, 2010). En este contexto, adquiere relevancia la Contabilidad de Costos como una herramienta que aporta información útil para el análisis y el control de la gestión que puede repercutir en la minimización de costos, tiempos y mayor calidad de los productos en las empresas.

Hoy en día, las Pymes tienen una importancia económica para el país porque contribuye a la generación de riqueza y creación de empleos, ya que éstas representan en la mayor parte del mundo más de $95 \%$ del número total de empresas, generando entre 60 y $70 \%$ del empleo y contribuyen entre 50 y $60 \%$ del Producto Interno Bruto (PIB) (OCDE, 2012). En el caso de México, las Pymes también son un elemento fundamental en favor del desarrollo eco- nómico y la creación de empleos. Consideran- do a las Microempresas en el Censo Económico realizado en el 2015 se observa que representan 99.8\% y generan 52\% del PIB (Producto Interno Bruto) del total de empresas (INEGI, 2016).

El principal reto de las Pymes es hacer frente al entorno competitivo en donde, los sistemas de costos juegan un papel fundamental (López y Marín, 2010). Con base en lo anterior, es necesario tomar en cuenta que el surgimiento de la tecnología de información y de las herramientas tecnológicas han modificado los procesos de administración contable y fiscal de las empresas obligando a la empresa a desarrollar nuevas estrategias en los sistemas de información contable, no sólo para adaptarse a las exigencias de la tecnología, sino también para el logro de toma de decisiones enfocadas a los mejores resultados, es por eso que la empresa debe contar con información de costos oportuna que le ayude a disminuir los errores, cumplir con las leyes fiscales, hacer las cosas diferentes y con ahorro de tiempo en la toma de decisiones.

Si bien es cierto que la mayoría de las Pymes conocen sus costos de logística, producción, comercialización y distribución en forma general, también es cierto que no analizan los costos indirectos de fabricación por carecer de sistemas de información gerencial, que permitan cruzar 
las fronteras de la estrategia competitiva, el posicionamiento estratégico y alcanzar la competitividad y lo más importante medir si generan o no valor agregado para los inversionistas (Aguirre y Armenta, 2012). El mismo autor menciona que las Pymes a fin de determinar sus eficiencias de costos deben conocer la información de tiempos por cada proceso productivo, por producto, concentración y forma de presentación. Sin embargo, estas empresas no se tienen claro la aplicación de los sistemas de costos directos o variables hacia los procesos de fijación de precios y más aún si son controlados por el estado.

Desde el punto de vista gerencial, las Pymes muestran una diversidad de deficiencias, entre las que está la utilización limitada de información de costos en la toma de decisiones. Para la mejora de la gestión empresarial de estas empresas, es necesario hacer uso de métodos y estrategias que les permita aprovechar la información que ayude a la gerencia a contar con información de costos pertinente y oportuna para aplicar estrategias efectivas al momento de la toma de decisiones. Partiendo de lo antes expuesto, el objetivo de la presente investigación es determinar con base en las opiniones de los empresarios Pymes, la importancia de los costos y sus beneficios en la gestión empresarial de las Pymes, con el fin de determinar la necesidad de diseñar un sistema de información de costos que ayude en la toma de decisiones, con el propósito de lograr una gestión empresarial encaminada a la competitividad.

\section{Revisión de literatura}

Las pequeñas y medianas empresas (Pymes), representan una importante fuente de trabajo en México, ya que junto con las microempresas generan casi el $80 \%$ de los empleos. Sus sencillas estructuras administrativas les conceden un mayor margen de maniobra para incursionar en proyectos administrativos innovadores, enfrentando un menor riesgo. Sin embargo, en las Pymes es práctica común que, con la idea de incrementar los márgenes de contribución, se tomen decisiones relacionadas con la reducción arbitraria de costos sin considerar que estos 'recortes' amenazan en su generalidad la calidad o a la operación de la entidad, principalmente porque se enfocan a los síntomas y no las causas reales de los costos (Backer, Jacobsen y Ramírez, 2006).

La contabilidad deriva información indispensable para la administración y el desarrollo del sistema económico por ello, las empresas y en especial en las Pymes, recae la necesidad de 


\section{http://revistainvestigacionacademicasinfrontera.com}

implementar sistemas de información contable de tal forma que sea mucho más fácil la toma de decisiones para administradores, propietarios, inversionistas, proveedores, entre otros (García, Marín y Martínez, 2006). La contabilidad general incluye entre las fases de su procedimiento, la contabilidad de costos; a través de ella se registran, analizan e interpretan los detalles de los costos de material, mano de obra y gastos indirectos, necesarios para producir y vender artículos; con objetivos definidos encauzados a planificar, controlar y tomar decisiones (Chang, Alba, González, López y Moreno, 2016).

Castillo (2015), manifiesta que para lograr el fortalecimiento y crecimiento de las actividades de las Pymes, es necesario contar con un soporte técnico en los sectores administrativos y contables con el objetivo de gestionar sus procesos y sus negocios, más aun cuando se trata del sector industrial, en donde la gestión de costos de producción puede ser considerada como una de las herramientas de base para la administración. Siendo así, podemos considerar a la gestión de costo como un pilar fundamental en la gestión empresarial de una Pyme debido a que ella proporciona un nivel de información esencial para la toma de decisiones, y por consiguiente, la carencia de un eficaz sistema de gerenciamiento de costos puede limitar la competitividad y crecimiento de una empresa o, en el peor de los casos, puede llevar al fracaso de la misma.

Como consecuencia de lo anterior, la teoría de la contabilidad ha evidenciado una doble relación entre lo administrativo y lo contable, que corresponde a momentos históricos distintos de la disciplina. Un primer estado demuestra que lo administrativo determina o influencia lo contable, mientras que uno posterior ha evidenciado que lo contable ayuda a impulsar a las organizaciones (Hernández, 2006, pp. 206-208). Las organizaciones deberían contar con un sistema de información contable integral, configurado por una contabilidad financiera y una contabilidad de costos, que nutriera a sus sistemas de control de gestión y permitiera a sus usuarios internos beneficiarse de información oportuna, pertinente y comparable a fin de que estos pudieran traducir las estrategias en resultados empresariales (Chacón, 2011). De allí que la contabilidad y 
los costos se convierten en el elemento básico de las finanzas de una empresa, pues, mediante su adecuada utilización, puede realizarse una gestión eficiente de los activos de la empresa, representados por su valor económico (Vaivio y Sirén, 2010).

En un ambiente de globalización, los sistemas de información contable y de costos son un concepto fundamental dentro de los procesos de ventaja competitiva, por ello cada vez nacen más estudios teóricos y empíricos que profundizan sus características (Castillo y Villareal, 2014). Una de las estrategias empresariales comunes utilizadas por la empresa, son los indicadores de rentabilidad, lucratividad o rendimiento que pretenden medir la efectividad con que la administración controla los costos y gastos operacionales para convertir las ventas en utilidades (Ortiz, 2004). La información que generan los costos es de vital importancia para la contabilidad de gestión en el sistema de información contable, porque de forma conjunta establecen su importancia para el establecimiento de la estrategia y desarrollo de las ventajas competitivas, su incidencia en la rentabilidad de las empresas y en la toma de decisiones (Buelvas y Mejía, 2015).

Hamel (2008), establece que las características de la gestión e innovación administrativa giran alrededor de los procesos del negocio (compra, producción, comercialización, despacho de pedidos, servicio al cliente, etc.). En ese sentido, estar bien informados conduce a una eficiente gestión empresarial, lo cual implicaría mayores niveles de competitividad. En este orden de ideas, Aktouf (2009), señala que tomar buenas decisiones es mejorar la gestión, obtener mayores ganancias; y decidir mejor como puede la gerencia, estar informados de la manera más perfecta posible y disponer de los métodos, instrumentos y estrategias que procesen dicha información para la mejora de la toma de decisiones en la gestión empresarial. Por este motivo, el entorno en el que se desenvuelven la mayoría de organizaciones empresariales obliga a sus dirigentes a incrementar la flexibilidad operativa de su organización y adaptarse con rapidez, a los cambios y paradigmas contables con el propósito de lograr la mayor eficiencia o performance mediante adecuados sistemas de información basada en los costos que ayuden a la gerencia a realizar una toma de decisiones efectiva (Buelvas y Mejía, 2015).

Tabitha y Ogungbade, (2016), mencionan que la era de la industrialización trajo consigo el desarrollo de un grupo importante de técnicas contables que se han originado en el seno de las 
Año 11.

Revista de Investigación Núm. 28 Académica sin Frontera ISSN: 2007-8870

\section{http://revistainvestigacionacademicasinfrontera.com}

actividades de manufactura, las cuales siguen vigentes en la actualidad y se han desplazado como prácticas en la agroindustria, lo que ha permitido el desarrollo de la contabilidad de costo y la práctica de costeo que ha ganado importancia y ha sido muy relevante para la gestión de costo, así como para la gestión empresarial y la ventaja competitiva en compañías de transporte, producción y distribución de países tales como Estados Unidos. La mayoría de las técnicas de costeo creadas en el siglo XIX, son actualmente conocidas y utilizadas como "técnicas tradicionales de costos", entre las cuales se pueden mencionar el costeo estándar, el costeo variable, el costeo por absorción, los costos marginales entre otros, las cuales han recibido muchas críticas, como por ejemplo: que han perdido relevancia debido a la innovación y el dinamismo de los entornos empresariales actuales, lo cual ha dado como resultado el desarrollo de nuevas técnicas como el costeo por actividad, el Cálculo del Costo del Ciclo de Vida, el Sistema Just in Time, entre otros (Nasieku \& Ogungbade, 2016).

Kaplan y Norton (2012), señalan la importancia de implementar una estrategia visionaria junto a excelentes procesos operacionales, mejorar la calidad, reducir costos y tiempos del proceso. Así mismo, Hamel (2008), establece que las características de la gestión e innovación administrativa giran alrededor de los procesos del negocio (compra, producción, comercialización, despacho de pedidos, servicio al cliente, etc.). En ese sentido, estar bien informados conduce a un eficiente desempeño, lo cual implicaría mayores niveles de competitividad. En este orden de ideas, Aktouf (2009), señala que tomar buenas decisiones es administrar mejor, obtener mayores ganancias; y decidir mejor es estar informados de la manera más perfecta posible y disponer de los métodos, instrumentos y estrategias que procesen dicha información.

Por este motivo, el entorno en el que se desenvuelven la mayoría de organizaciones empresariales obliga a sus dirigentes a incrementar la flexibilidad operativa de su organización y adaptarse con rapidez, a los cambios y paradigmas contables con el propósito de lograr la mayor eficiencia o 
performance mediante adecuados sistemas de información basada en los costos que ayuden a la gerencia a realizar una toma de decisiones efectiva (Buelvas y Mejía, 2015).

Recientemente, Lafuente y Paez (2018), realizaron una revisión sobre las prácticas de gestión de costos utilizadas en las Pymes de Paraguay, con el fin de identificar cuáles son las prácticas actuales en la gestión de costo, determinar las barreras que puedan estar impidiendo la adopción de las técnicas modernas de gestión de costeo, como así también evaluar la percepción por parte de los encargados del sector sobre los posibles beneficios con la adopción de los modelos de gestión integral de costeo. Los resultados obtenidos por los autores, les revelaron que como

práctica más difundida en el sector se encuentran las herramientas empíricas; desarrollas a medida de la necesidad de cada empresa, el escaso nivel de utilización de sistemas informáticos de costeo y la escasa difusión y/o conocimiento por parte del sector analizado de las nuevas herramientas de gestión de costos. Entre las barreras más resaltantes encontradas mencionan: la dificultad de identificar a los generadores de costos y la calidad de los servicios de los consultores, por último, se observa una buena apertura de parte de los directivos de las empresas encuestadas sobre los beneficios que podrían acarrear la aplicación de las herramientas de gestión en cuanto a la toma de decisiones basada en datos.

\section{Metodología}

\section{Diseño de la investigación}

Este estudio se basa en una investigación de campo bajo un enfoque metodológico descriptivo de corte transversal con el objetivo de determinar con base en las opiniones de los empresarios Pymes, la importancia de los costos y sus beneficios en la gestión empresarial.

\section{Elección y tipo de muestra}

El universo seleccionado para esta investigación se eligió bajo el mismo criterio de estratificación de las empresas utilizado por el INEGI para los censos económicos 2016 de México que fueron obtenidas en noviembre del 2017 y acordados por los integrantes de la investigación, al cual se le 


\section{http://revistainvestigacionacademicasinfrontera.com}

anexarán otros criterios tales como: Pymes del sector comercio afiliados a CANACO de la ciudad de Hermosillo, Sonora, México, con una fuerza laboral entre 10 y 250 trabajadores, una antigüedad mínima de 10 años en la actividad. El universo de la población son 1270 empresas de las cuales 796 corresponden Pymes con las características elegidas para este estudio. El total de la muestra es de 116 empresas, la cual se fue elegida con la fórmula para una población finita y un muestreo sin reemplazo siguiente:

$$
\begin{gathered}
\mathrm{n}=\frac{\mathrm{NZ} \mathrm{Z}^{2} \mathrm{pq}}{\left(\mathrm{Z}^{2} \mathrm{pq}\right)+\left[\mathrm{d}^{2}(\mathrm{~N}-1)\right]} \\
\mathbf{n}=\mathbf{1 1 5 . 7 6 8 7} \approx \mathbf{1 1 6} \text { empresas }
\end{gathered}
$$

\section{Instrumento de recolección de datos}

Para poder realizar la comparación de las respuestas y por ende su categorización y generalización, se utilizará la entrevista abierta apoyada por un cuestionario estructurado donde se analizan tres variables: Variable 1; Influencia de la Información que ofrecen los costos para la toma de decisiones. En los resultados obtenidos se analiza el tipo de información que ofrece los costos y cómo influye en la toma de decisiones dentro de la gestión empresarial de las Pymes Y, Variable 2; Principales actividades de gestión empresarial. Actividades donde es importante contar con información oportuna de costos para realizar una toma de decisiones efectiva por parte de la gerencia de la Pyme. 
Para estimar la confiabilidad del instrumento aplicado, se hizo uso del programa IBM SPSS (Paquete Estadístico para las Ciencias Sociales, por sus siglas en inglés), para obtener el Alfa de Cronbach en cada una de los items, obteniendo en lo general un coeficiente de .900 . Posteriormente, con la finalidad de detectar errores de diseño y la factibilidad del instrumento, se llevó a cabo una prueba piloto con un mínimo de empresarios con las características de la muestra elegida, para de esta manera realizar los ajustes necesarios en las mismas y así aunada a la prueba de fiabilidad realizada a través del programa SPSS alcancen un elevado grado de confiabilidad. Las encuestas fueron aplicadas directamente a los responsables de las empresas seleccionadas, que permita obtener información que sea verídica y confiable. La información recopilada, organizada y ordenada, será capturada en el programa estadístico SPSS (Statistical Package for the Social Sciences), para su valoración e interpretación.

\section{Presentación, análisis e interpretación de resultados}

Tras analizar los datos obtenidos por medio de los estudios exploratorios, a continuación, se presentan los resultados más relevantes del trabajo de investigación basado en el objetivo central de la investigación dentro de la fase exploratoria de la investigación, tomando en consideración las dos variables de trabajo.

\subsection{Variable 1, Influencia de la Información que ofrecen los costos para la toma de decisiones. En los resultados obtenidos se analiza el tipo de información que ofrece los costos y cómo influye en la toma de decisiones dentro de la gestión empresarial de las Pymes.}


Núm. 28

ISSN: 2007-8870

\section{http://revistainvestigacionacademicasinfrontera.com}

Imagen 1: Se lleva a cabo por parte de la Pyme una contabilidad de costos que se ocupa de la clasificación, acumulación, control y asignación de costos

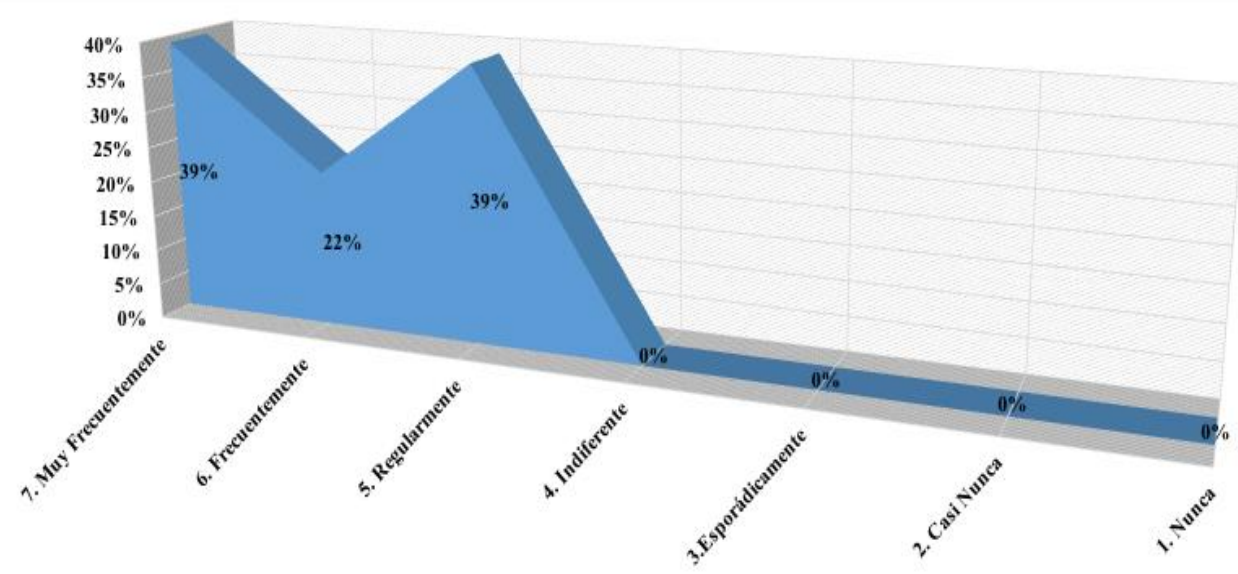

Fuente: Elaboración propia con base en los resultados obtenidos.

La Imagen 1; muestra que de acuerdo a las opiniones de los empresarios hay una coincidencia del $39 \%$ en referencia a que muy frecuentemente y regularmente se lleva a cabo por parte de las Pymes una contabilidad de costos que se ocupa de la clasificación, acumulación, control y asignación de costos, el 22\% opina que frecuentemente se lleva a cabo. Los resultados muestran que, de acuerdo a las opiniones de los empresarios, al parecer están conscientes que es importante llevar a cabo una contabilidad de costos. 
Imagen 2: La información que generan los costos suministra tanto medidas objetivas de operaciones pasadas como uso de estimados subjetivos sobre decisiones futuras

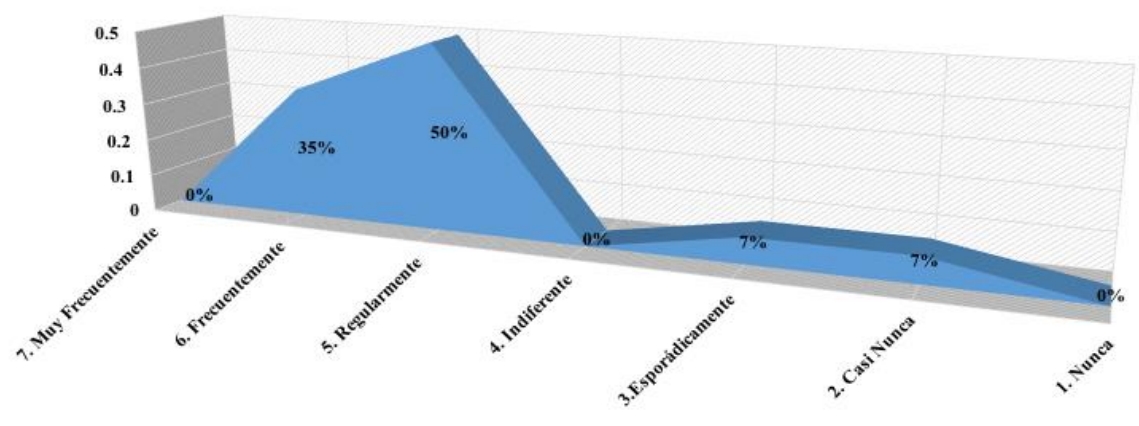

Fuente: Elaboración propia con base en los resultados obtenidos.

La Imagen 2; muestra que de acuerdo a las opiniones del 50\% de los empresarios, regularmente la información que generan los costos suministra tanto medidas objetivas de operaciones pasadas como el uso de estimados subjetivos sobre decisiones futuras para el empresario Pyme, el 35\% opina que frecuentemente hay una coincidencia, el $7 \%$ de los expertos opina que esporádicamente o casi nunca la hay. Los resultados muestran que, de acuerdo a las opiniones de los empresarios, al parecer la información que genera los costos les permite tomar decisiones objetivas.

Imagen 3: Los costos son medidos en términos reales, esto es con el poder adquisitivo y en términos monetarios, es decir, en valores nominales

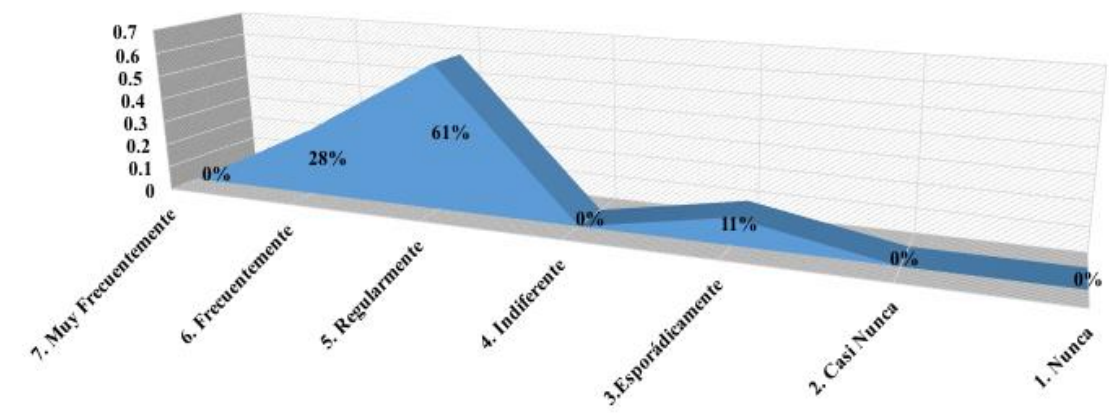

Fuente: Elaboración propia con base en los resultados obtenidos. 


\section{http://revistainvestigacionacademicasinfrontera.com}

La Imagen 3; muestra que de acuerdo al $61 \%$ de las opiniones de los empresarios de la mestra analizada, regularmente los costos son medidos en términos reales, esto es con el poder adquisitivo y en términos monetarios, es decir, en valores nominales dentro de las Pymes, el 28\% menciona que son medidos frecuentemente y el $11 \%$ es medido esporádicamente. Lo anterior, muestra que, de acuerdo a las opiniones de los empresarios, al parecer los valores nominales que genera el poder adquisitivo en términos monetarios es importante para la empresa.

Imagen 4: La información de los costos permite generar un mayor control sobre la calidad y eficiencia de las diferentes áreas de la empresa

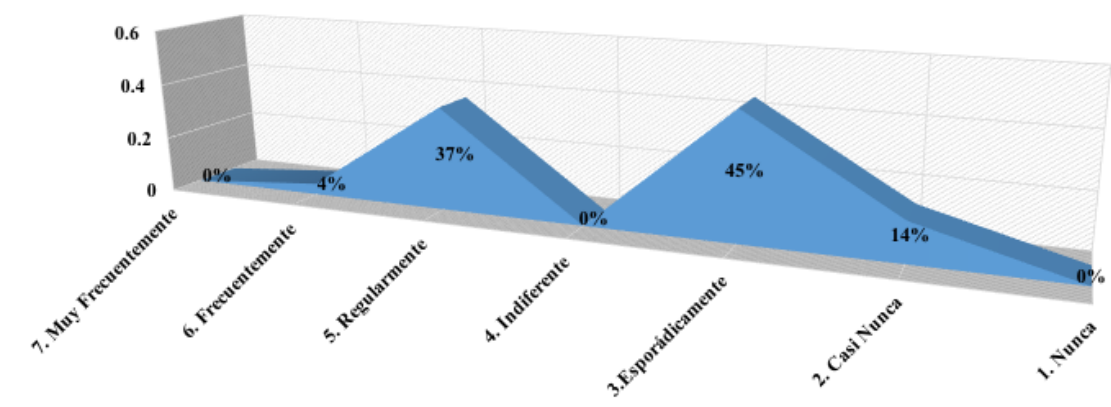

Fuente: Elaboración propia con base en los resultados obtenidos.

La Imagen 4; muestra que de acuerdo al 45\% de las opiniones de los expertos, esporádicamente la información de los costos permite generar un mayor control sobre la calidad y eficiencia de las diferentes áreas de la empresa, el 37\% opina que regularmente le permite, el 14\% casi nunca y el $4 \%$ frecuentemente. Lo anterior, muestra que, de acuerdo a las opiniones de los expertos, al parecer el empresario Pyme está consciente de que la información de los costos le permite mayor control de operación para lograr sus objetivos y metas. 
Imagen 5: Es necesario implantar un sistema de costos en la empresa para poder vincularla al sistema económico

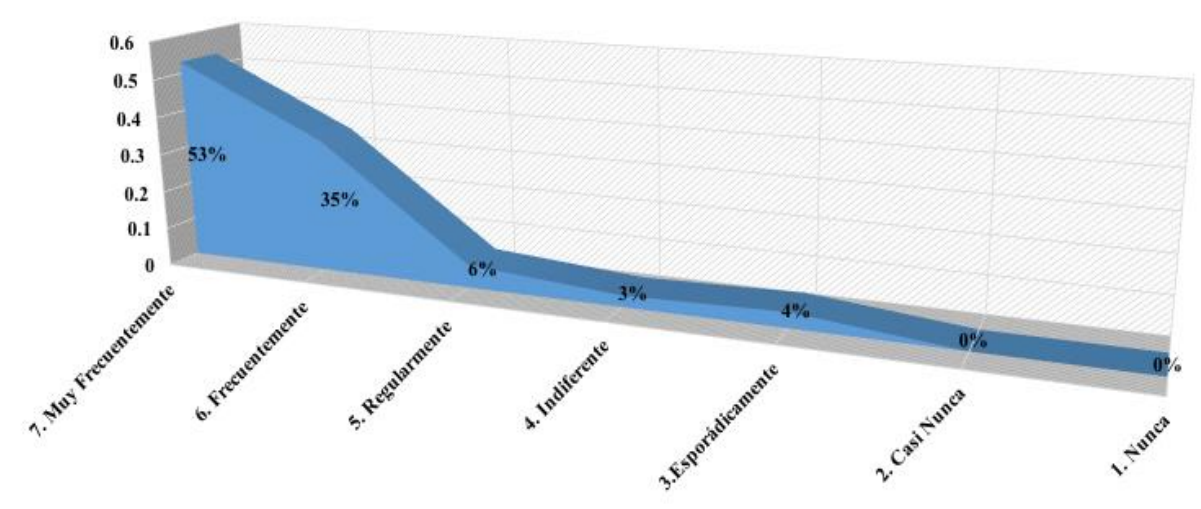

Fuente: Elaboración propia con base en los resultados obtenidos.

La Imagen 5; muestra que de acuerdo a las opiniones del 53\% de los empresarios de la muestra analizada, muy frecuentemente es necesario que la Pyme implante un sistema de costos para poder vincularla al sistema económico, el $35 \%$ opina que frecuentemente, el $4 \%$ esporádicamente y el $3 \%$ indiferente. Los resultados muestran que de acuerdo a las opiniones de los empresarios es importante que la Pyme pueda implantar un sistema de costos, al parecer están conscientes de que esto ayuda a la empresa a lograr una vinculación interna de sus áreas y mayor control financiero.

Imagen 6: Utiliza indicadores financieros para evaluar los resultados de las diferentes áreas de la empresa

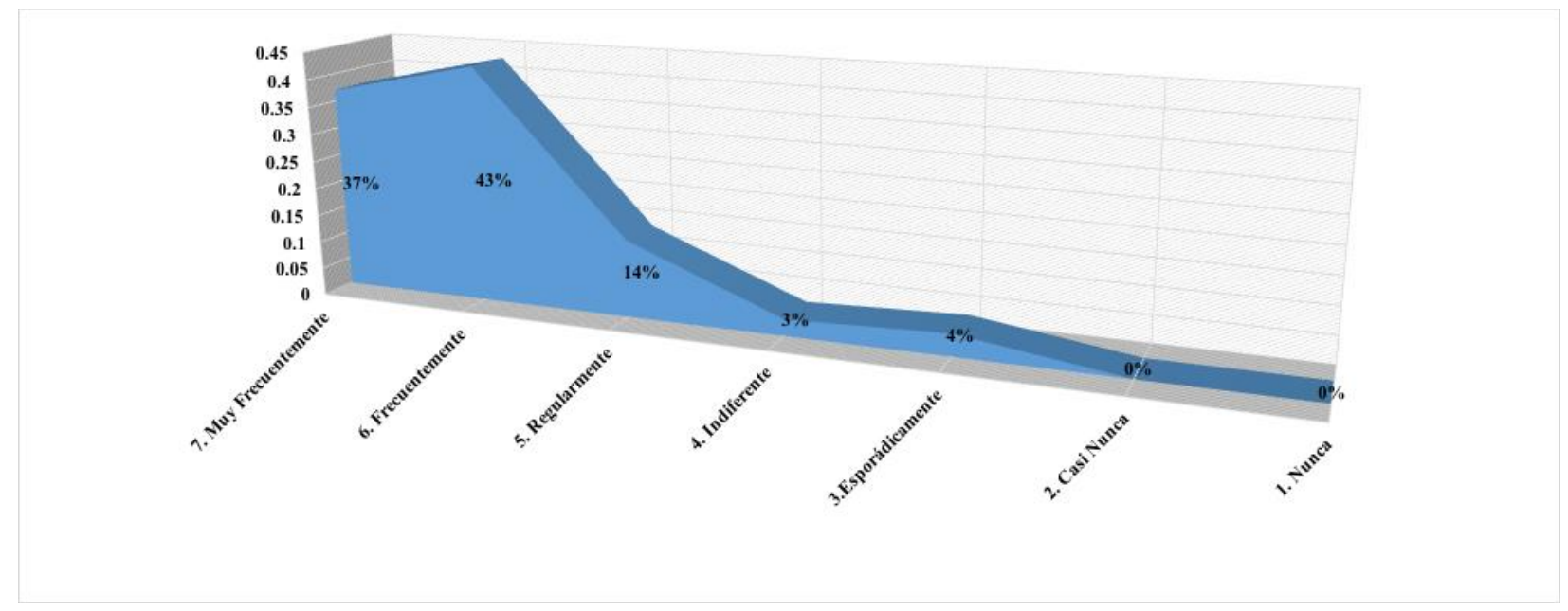

Fuente: Elaboración propia con base en los resultados obtenidos. 


\section{http://revistainvestigacionacademicasinfrontera.com}

La Imagen 6; muestra que de acuerdo a las opiniones del $43 \%$ de los empresarios, frecuentemente la Pyme utiliza indicadores financieros para evaluar los resultados de las diferentes áreas de la empresa, el $37 \%$ opina que muy frecuentemente, el 14\% regularmente, el $4 \%$ esporádicamente y el 3\% indiferente. Los resultados muestran la importancia que tiene para los empresarios que sus negocios utilicen indicadores financieros para evaluar resultados, al parecer ellos están conscientes de que esto permite a la empresa conocer la eficiencia de las áreas de la empresa.

Imagen 7: La información de costos le permite analizar, planear, controlar, y tomar decisiones relativas a ventas, recursos humanos, base para datos, productos, utilidades, etc.

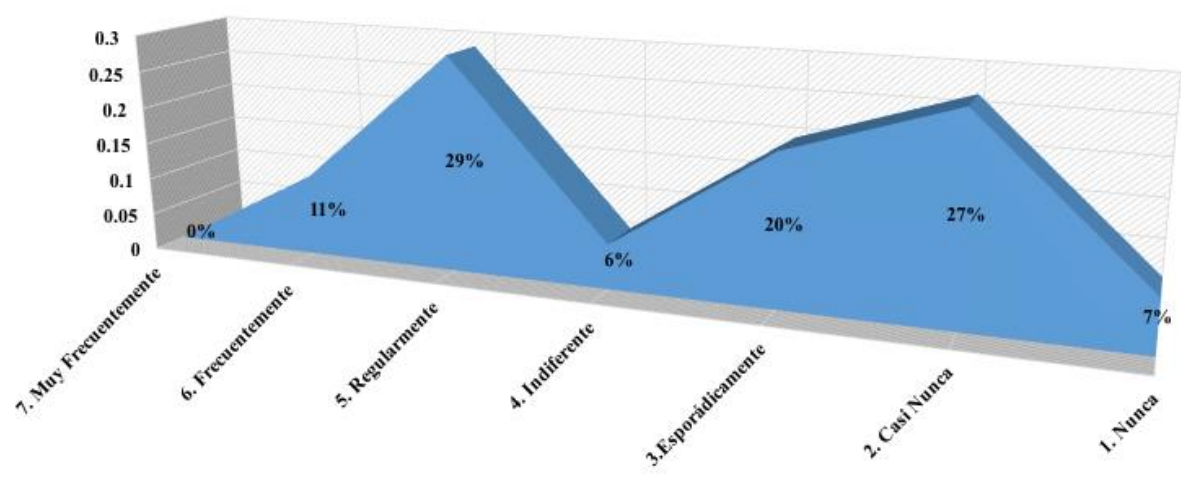

Fuente: Elaboración propia con base en los resultados obtenidos.

La Imagen 7; muestra que de acuerdo a las opiniones del 29\% de los empresarios, regularmente para la Pyme la información de costos le permite analizar, planear, controlar, y tomar decisiones relativas a ventas, recursos humanos, base para datos, productos, utilidades, etc., el 27\% opina que casi nunca, el $20 \%$ esporádicamente, $11 \%$ frecuentemente y $7 \%$ nunca le permite. Los resultados muestran que de acuerdo a la opinión de los empresarios, la información de costos es importante para la toma de decisiones y, posiblemente no cuentan con la información a tiempo o no saben utilizarla. 
Imagen 8: La información de costos de la empresa le llega con puntualidad y exactitud al momento de tomar decisiones

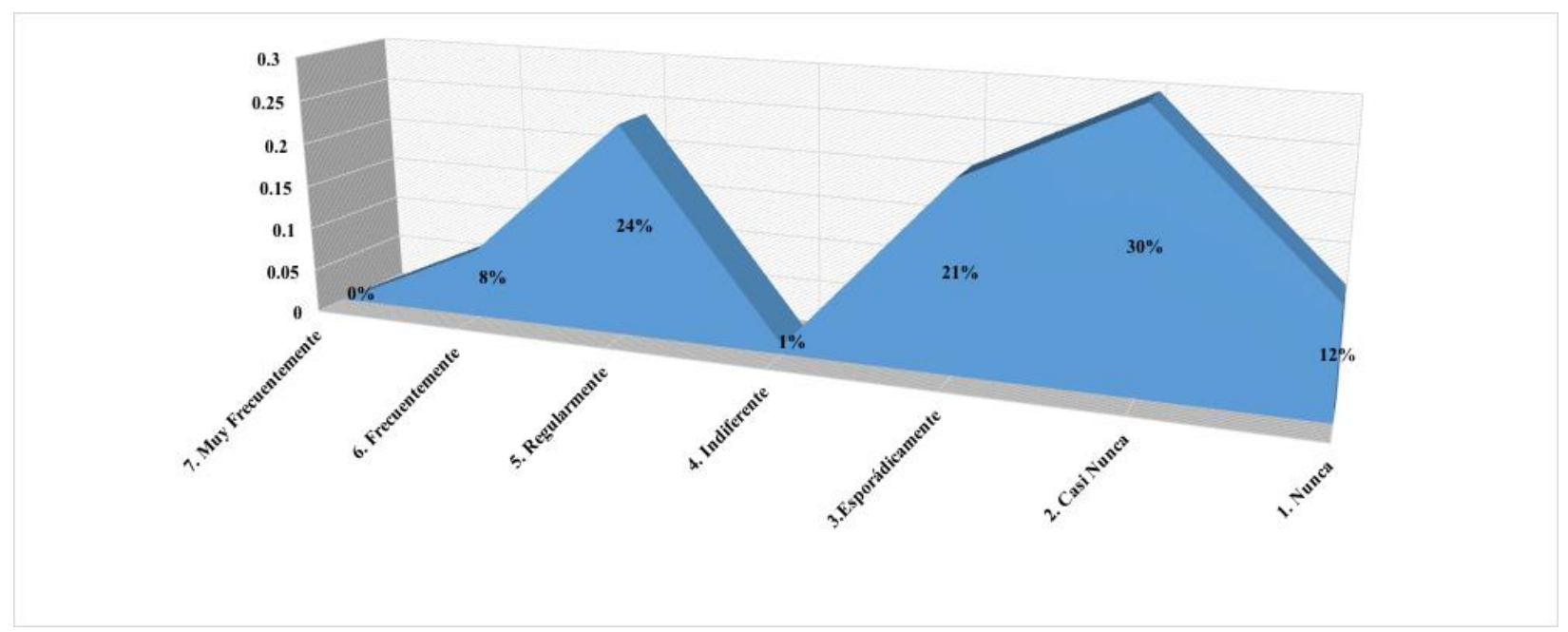

Fuente: Elaboración propia con base en los resultados obtenidos.

La Imagen 8; muestra que de acuerdo a las opiniones del 30\% de los empresarios, casi nunca la información de costos de la empresa le llega al empresario con puntualidad y exactitud al momento de tomar decisiones, el $24 \%$ opina que regularmente le llega, el $21 \%$ opina que esporádicamente le llega, el $12 \%$ nunca le llega y el $8 \%$ frecuentemente. Los resultados muestran que para los empresarios de las Pymes de la muestra analizada, la información de costos no es oportuna al momento de tomar decisiones, esto al parecer puede ser problema en los sistemas de información o los canales de comunicación de la empresa. 
Núm. 28

ISSN: 2007-8870

\section{http://revistainvestigacionacademicasinfrontera.com}

Imagen 9: La empresa requiere contar con innovación tecnológica para eficientar la información de costos

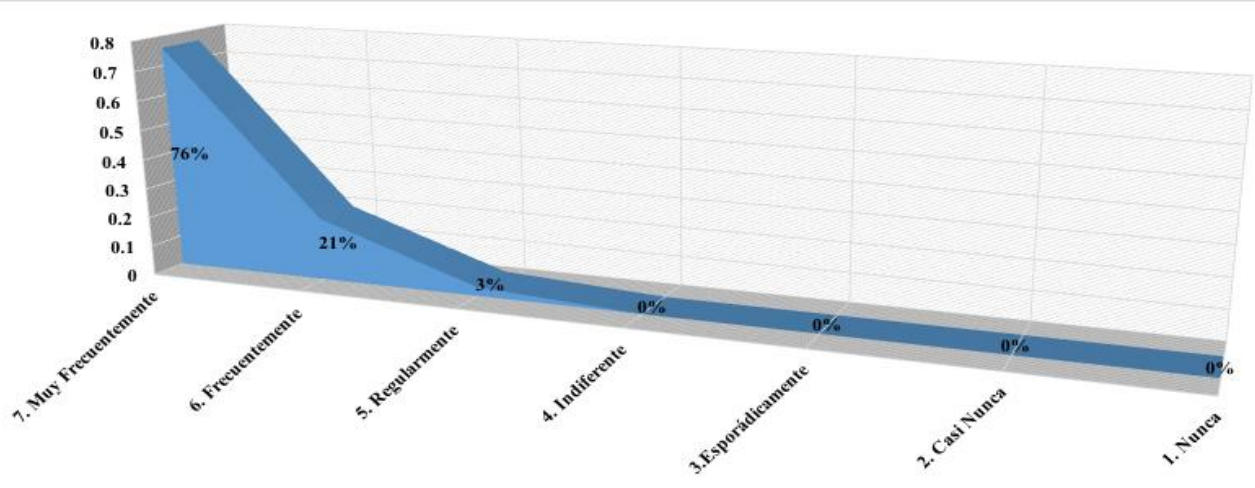

Fuente: Elaboración propia con base en los resultados obtenidos.

La Imagen 9; muestra que de acuerdo a las opiniones del 76\% de los empresarios, muy frecuentemente la Pyme requiere contar con innovación tecnológica para eficientar la información de costos, el $21 \%$ opina que frecuentemente y el 3\% regularmente. Los resultados muestran que, de acuerdo a las opiniones de los empresarios Pymes, requieren posiblemente una actualización en el tema de innovación tecnológica.

Imagen 10: Su empresa requiere contar con un modelo o técnica de Contabilidad de Costos que genere información más oportuna para la toma de decisiones

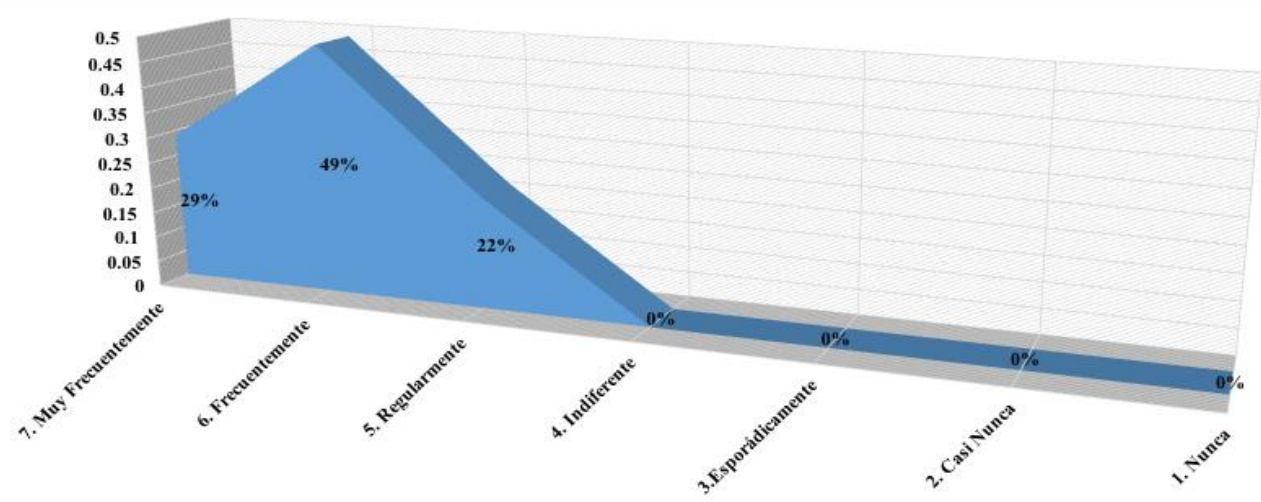

Fuente: Elaboración propia con base en los resultados obtenidos. 
La Imagen 10; muestra que de acuerdo a las opiniones del 49\% de los empresarios, frecuentemente la Pyme requiere contar con un modelo o técnica de Contabilidad de Costos que genere información más oportuna para la toma de decisiones, el 29\% opina que muy frecuentemente y el $22 \%$ regularmente. Los resultados muestran la importancia que tiene para los empresarios de las Pymes cuenten con un modelo o técnica de Contabilidad de Costos para que puedan contar con información oportuna al momento de tomar decisiones.

Variable 2; Principales actividades de gestión empresarial. Actividades donde es importante contar con información oportuna de costos para realizar una toma de decisiones efectiva por parte de la gerencia de la Pyme.

Imagen 11: Actividades de gestión empresarial que requieren información de costos para la toma de decisiones

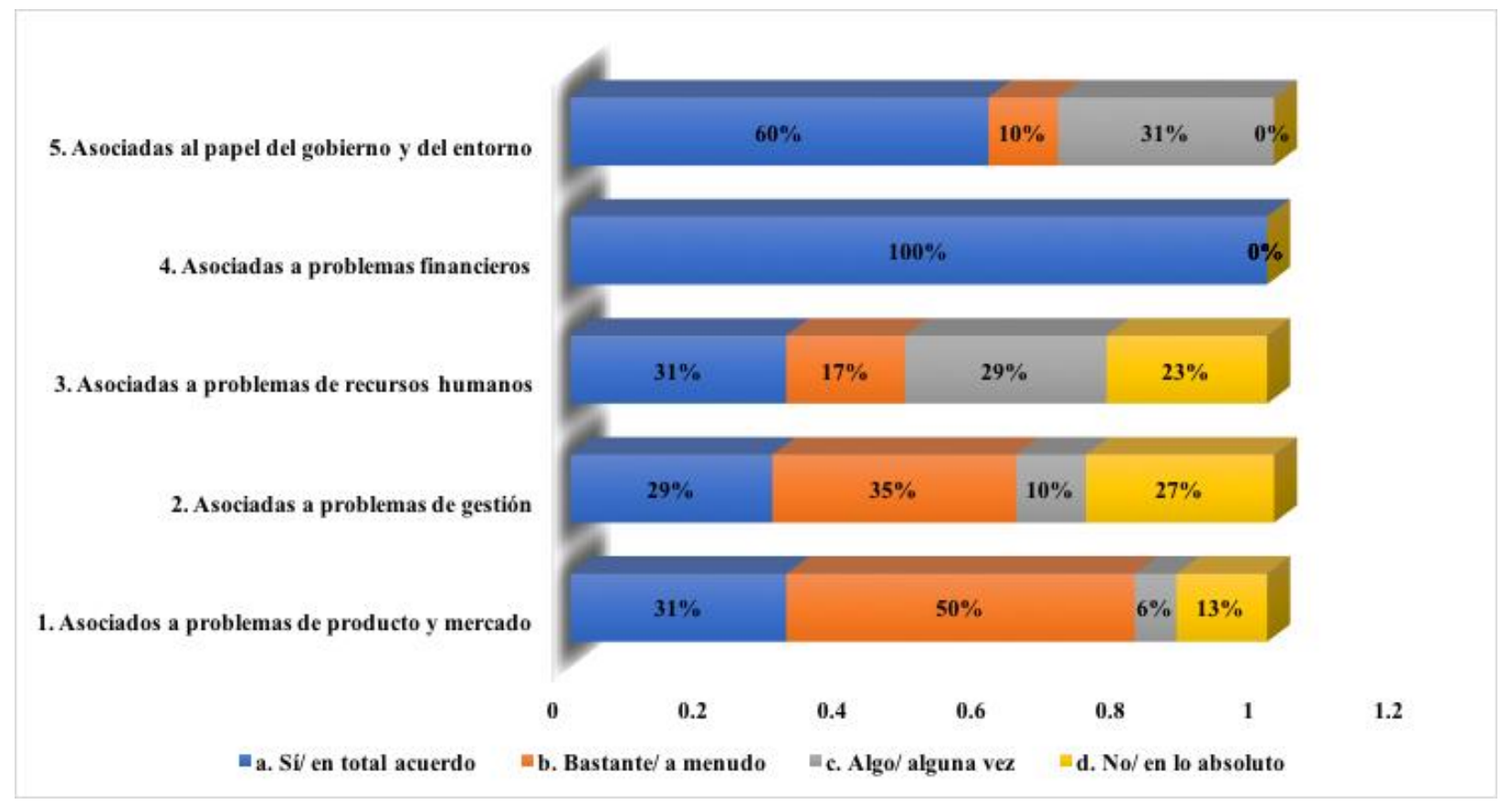

Fuente: Elaboración propia con base en los resultados obtenidos.

La Imagen 11, muestra que de acuerdo al 100\% de las opiniones de los expertos opinan estar en total acuerdo de que la principal actividad que requiere información oportuna para la toma de decisiones por parte del empresario Pyme es la asociada a problemas financieros. En segundo lugar, un $60 \%$ de los expertos opinan que están en total acuerdo de que la información asociada al papel del gobierno y del entorno. En tercer orden, el $50 \%$ de los expertos opinan que la 


\section{http://revistainvestigacionacademicasinfrontera.com}

información asociada a problemas de producto y mercado. En cuarto orden, un $35 \%$ de los expertos opinan que a menudo son las asociadas a problemas de gestión. En último lugar son las asociadas a problemas de personal.

\section{Conclusiones e implicaciones sociales y empresariales}

Dentro del modelo de toma de decisiones la contabilidad de costos, ayuda a la gerencia para que la decisión sea mejor, de acuerdo con la calidad de la información que se posea. En toda organización diariamente se toman decisiones, unas son rutinarias, como contratar un nuevo empleado; otras no la son, como introducir o eliminar una línea de producto, ambas requieren adecuada información. El empresario Pyme debe contar con métodos alternativos de costeo, y herramientas fundamentales en la toma de decisiones como son el punto de equilibrio, el modelo costo-volumen-utilidad, los problemas comunes en la toma de decisiones y la determinación de precios, que les permitirán comprender y analizar cada una de sus aplicaciones, que serán de gran utilidad en la tarea de gerenciar y sobre todo de tomar decisiones asertivas y positivas que lleven a la gestión empresarial a una ventaja competitiva.

Los principales resultados obtenidos en la presente investigación, se pone de manifiesto que de acuerdo a las opiniones del gerente de las Pymes de la muestra analizada, consideran positiva los costos para la obtención relevante de información para la toma de decisiones que ayude en la gestión empresarial. Por otro lado, consideran que la utilización de un sistema de gestión de costos podría mejorar las medidas de desempeño económico y operativo de la empresa, lo cual deja entrever la percepción de los encuestados sobre las herramientas de costo como una herramienta de control de resultados y no como una herramienta de gestión empresarial.

A modo de conclusión final y tomando en cuenta los planteamientos anteriores, cabe resaltar que dentro del modelo de toma de decisiones la contabilidad de costos, ayuda a la gerencia para que 
la decisión y establecimiento de estrategias de gestión sea mejor, de acuerdo con la calidad de la información que se posea. Por otro lado, la percepción de la importancia de los costos y beneficios en la gestión empresarial aquí planteados, debe extenderse a otros puntos no analizados, tales como las características comparativas de cada uno de los factores de éxito o fracaso que otorgan los sistemas de información de costos al momento de la creación de estrategias de gestión por parte de las Pymes en razón a su giro económico.

Estos resultados comprueban el objetivo planteado en la investigación donde se hace ver desde la perspectiva del empresario Pyme, la importancia de los costos y sus beneficios en la gestión empresarial de las Pymes. La realización de este proyecto se considera viable ya que Sonora, cuentan con un sector económico muy dinámico en sus diferentes sectores, tanto el industrial, el comercial y el de servicios. Para Sonora, la Pyme ocupa un lugar preponderante en cada uno de ellos. En esta investigación se pretende demostrar que existe una alta necesidad de realizar más trabajos de investigación relacionados con el tema, con enfoques específicos por sector, región y actividad, así como darlos a conocer a las cámaras de las empresas y a las asociaciones de profesionistas para que difundan los contenidos entre los empresarios Pymes.

\section{Referencias bibliográficas}

Aguirre Choix, R. y Armenta Vázquez, C. E. (2012). La importancia del control interno en las pequeñas y medianas empresas en méxico. Revista El Buzón de Pacioli, Año XII Número 76 , pp. 1-17.

Aktouf, O. (2009). La administración: entre tradición y renovación. Cali: Coedición, Universidad del Valle, Universidad Libre, Artes gráficas del Valle.

Backer, A. C., Jacobsen, M. y Ramírez, J. (2006). Contabilidad de costos: un enfoque administrativo para la toma de decisiones. Editorial McGraw Hill, México.

Buelvas-Meza, C.G. y Mejía-Alfaro, G.E. (2015). El papel de la contabilidad de gestión en el sistema de información contable y su incidencia en la rentabilidad de las empresas. Revista panorama económico, Vol. 22, pp. 91-108.

Castillo Pérez, A.A. (2015). Importancia de la calidad de la información de los sistemas informáticos contables en las empresas. In Crescendo. Ciencias Contables y Administrativas, 2(2), pp. 123-141 
Año 11.

Revista de Investigación

Núm. 28

Académica sin Frontera

ISSN: 2007-8870

\section{http://revistainvestigacionacademicasinfrontera.com}

Castillo, C.; Villareal, J. (2014). Los Sistemas de Información Contable y el Balanced Scorecard en las pymes industriales de Pasto.

Chacón, Galia B. (2011). La contabilidad de costos en el sistema de información contable de las PyME del estado Mérida Actualidad Contable Faces [en linea] 2011, 14 (Enero-Junio): [Fecha de consulta: 25 de junio de 2017] Disponible en:<http://www.redalyc.org/articulo. oa? $\mathrm{id}=25720061003>$ ISSN 1316-8533.

Chang, L., Alba, M., González, N., López, P. y Moreno, M. (2016). La importancia de la contabilidad de costos. [En línea], disponible en http://www.itson.mx/publicaciones/pacioli/documents/no60/costos.pdf

García, D., Marín, S., y Martínez, F. (2006): La contabilidad de costos y rentabilidad en la PYME. Contaduría y Administración, México, abril-junio, no. 218, pp. 39-50.

Hamel, G. (2008). El Futuro de la Administración. Bogotá: Editorial Norma S.A.

Hernández-Chaustre, S. A. (2006). La pyme en Latinoamérica. En Sergio Alberto HernándezChaustre; Sergio Calderón-Acevedo; Jorge Humberto Botero; Enrique Barriga; Margoth García; Juan Manuel Lesmes; Mauricio Jaramillo; Gustavo Aristizábal; Gustavo Ardila; Camilo Téllez; Claudia Vega; Marcial del Castillo; Natalia Jiménez \& Maribeth Murphy. El gran libro de las pymes - Información Financiera. Bogotá: Casa Editorial El Tiempo. 22-27.

INEGI (2016), censos económicos 2014-16, recuperado de: http://www.inegi.org.mx/est/contenidos/espanol/proyectos/censos/ce2009/default.asp?s=e $\mathrm{st} \& \mathrm{c}=1422, \mathrm{~m}$,

Kaplan, R. y Norton, D. (2012). Execution Premium. Bogotá: Deusto.

Lafuente Velazquez, M. R. y Paez Von Schmeling, N.A. (2018). Gestión de costos en las Pymes. Un análisis en las industrias gráficas de Paraguay. Iberoamerican Journal of Indurstrial Engineering, Florianópolis, SC, Brasil, V.10, N.19, pp. 84-105.

López Mejía, M. R. y Marín Hernández, S. (2010). Los Sistemas de Contabilidad de Costos en la PyME mexicana Investigación y Ciencia, vol. 18, núm. 47, pp. 49-56 
Martínez, R., Charterina, J., y Araujo, A. (2010). Un modelo causal de competitividad empresarial planteado desde la VBR: capacidades directivas, de innovación, marketing y calidad. Investigaciones Europeas de Dirección y Economía de la Empresa, 16 (2), 165188.

Nasieku Tabitha, Ogungbade, Oluyinka Isaiah. (2016) Cost Accounting Techniques Adopted by Manufacturing and Service Industry within the Last Decade. Disponible en: http://www.magementjournal.info/iNTERNACIONAL Journal of Advances in Management and Economics (IJAME) ISSN:2278-3369

OCDE (2012). Top Barriers and Drivers to SME Internationalisation, Centre for Entrepreneurship, SME and Local Development, (2008-2010)

Ortiz, H. (2004). Análisis financiero aplicado y principios de administración financiera. Décima Segunda Edición, Departamento de Publicaciones, Universidad Externado de Colombia, Colombia.

Saavedra García, M., Milla Toro, S. y Sánchez, B.. (2013). Determinación de la competitividad de la PYME en el nivel micro: El caso de del Distrito Federal, México. FAEDPYME INTERNATIONAL REVIEW, Norteamérica, Vol. 2, No. 4, pp. 18-23.

Tabitha y Ogungbade, (2016). Gestión de costos en las Pymes - un análisis en las industrias graficas del Paraguay. Iberoamerican Journal of Indurstrial Engineering, Florianópolis, SC, Brasil, V.10, N.19, P. 84-105.

Vaivio, J., \& Sirén, A. (2010). Insights into method triangulation and "paradigms" in interpretive management accounting research. Management Accounting Research, 21(2),130-141.

\footnotetext{
Para citar este artículo

Martín Guillermo Durán Acosta, María Lizett Zolano Sánchez, Leticia Del Carmen Encinas Meléndrez. Importancia De La Gestión De Costos En Las PYMES Del Ector Servicios De Hermosillo, Sonora. RDIASF. Núm. 28 (2018). ISSN 20078870. Pp. XX-XX.
} 